\title{
Importancia de microlitiasis en ultrasonido de neoplasias de células germinales de testículo
}

\author{
Villalobos Gollás $\mathrm{M}^{*}$, Negrete Pulido $\mathrm{O}^{*}$, Mendez Probst $\mathrm{C}^{*}$, Fuentes Corona $\mathrm{R}^{* *}$, \\ Sotomayor de Zavaleta $\mathrm{M}^{*}$, Feria Bernal $\mathrm{G}^{*}$. \\ *Departamentos de Urología y **Radiología. Instituto Nacional de Ciencias Médicas y Nutrición \\ "Salvador Zubirán”, México D.F.
}

Actas Urol Esp. 2008;32(2):190-193

\section{RESUMEN}

\section{IMPORTANCIA DE MICROLITIASIS EN ULTRASONIDO DE NEOPLASIAS DE CÉLULAS GERMINALES DE TESTÍCULO}

Introducción: La microlitiasis testicular (MT) es un hallazgo poco frecuente en el ultrasonograma cuyo significado clínico no se ha definido. El presente estudio se realizó en pacientes con tumores de células germinales de testículo para analizar la relación entre MT, los hallazgos histológicos y las variables clinicas.

Material y métodos: Se incluyeron 57 pacientes sometidos a orquiectomía radical por neoplasia testicular germinal y se revisaron las variables clinicopatológicas y ultrasonográficas.

Resultados: Hubo 30 hombres (52,6\%) sin MT y 27 hombres con MT (48,27\%). Los pacientes con MT tuvieron mayor proporción de tumor germinal no seminomatoso (TGNS, 55,6\% vs 30\%, $p=0,05$ ), de cáncer testicular estadio II/III $(51,8 \%$ vs $16,7 \%$, p=0,005), de borde quirúrgico positivo $(18,5 \%$ vs $0 \%, p=0,021)$ y de invasión al cordón espermático $(14,8 \%$ vs $0 \%, p=0,048)$. No se encontró diferencia significativa en relación a otras variables histopatológicas.

Conclusión: En este estudio se demostró una asociación entre MT en tumores testiculares con el hallazgo de TGNS, estadio clínico avanzado, borde quirúrgico positivo e invasión al cordón.

Palabras clave: Tumores de testículo. Ultrasonido testicular. Histología. Microlitiasis testicular

\section{ABSTRACT}

IMPORTANCE OF MICROLITHIASIS IN TESTICULAR GERM CELL TUMOR ULTRASOUND

Introduction: Testicular microlithiasis (TM) is an infrequent finding in testicular ultrasound and its clinical importance has not been completely defined. We analyzed the ultrasounds of patients with testicular germ cell tumors in order to analyze the correlation between TM, histological findings and clinical variables.

Methods and materials: Fifty-seven patients with germ cell tumors and radical orchiectomy were included. Clinical, pathological, and echographic data were analyzed.

Results: TM was observed in 27 men (48.27\%) and was absent in 30 (52.6\%). Patients with TM had a greater likelihood of nonseminomatous germ cell tumors (NSGCT) vs seminomatous $(55.6 \%$ vs $30 \%$, $\mathrm{p}=0.05)$, stage II/III testicular cancer $(51.8 \%$ vs $16.7 \%, \mathrm{p}=0.005)$, positive surgical margins $(18.5 \% \mathrm{vs}$ $0 \%, p=0.021)$, and spermatic cord invasion $(14.8 \%$ vs $0 \%, p=0.048)$. No significant difference was found in respect to other histopathological variables.

Conclusion: This study showed that TM in testicular tumors is associated to NSGCT, advanced clinical stage, positive surgical margins, and spermatic cord invasion.

Keywords: Testicular tumors. Testicular ultrasound. Histology. Testicular microlithiasis. 
T a microlitiasis testicular (MT) es un hallazgo poco frecuente en el ultrasonograma. Su etiología y significado clínico aún son desconocidos; sin embargo, se encuentra asociada a múltiples patologías como: trastornos de la diferenciación sexual, criptorquidia, sub-fertilidad, enfermedades inflamatorias (granulomatosas), alteraciones vasculares (hemorragia con infarto, torsión testicular, calcificaciones vasculares), síndrome de inmunodeficiencia adquirida, neurofibromatosis y con coexistencia de cáncer testicular. Incluso los testículos sanos pueden tenerla.

El presente estudio se realizó en pacientes con tumor testicular maligno y se analizó su posible correlación con MT. Se revisaron los ultrasonogramas correspondientes a los hallazgos clínicos e histológicos.

\section{MATERIALES Y MÉTODOS}

Se revisaron los expedientes clínicos de pacientes con cáncer testicular (CaT) desde enero/1995 a junio/2006 y se identificaron 101 sometidos a 104 orquiectomías radicales por neoplasia testicular. Se excluyeron ocho que recibieron quimioterapia preoperatoria, 34 que no contaban con datos ultrasonográficos completos y 2 con linfoma testicular. Se incluyeron 57 pacientes que contaban con datos clínicopatológicos completos.

Se revisó el expediente clínico y radiográfico para determinar las variables clinicopatológicas y del ultrasonido (presencia de microlitiasis y su localización). Para la estratificación del estadio se unieron los pacientes en estadios II y III debido a la pequeña muestra de estos de forma independiente. Se evaluó si en algún momento del seguimiento requirieron tratamiento adyuvante y la incidencia de recurrencia de la enfermedad.

Se definió como microlitiasis testicular a tres o más focos ecogénicos con diámetro de 1-3 mm y distribución difusa en el parénquima testicular, ya sea unilateral o bilateral (Fig. 1).

El análisis estadístico entre los grupo se realizó con la prueba t de student. Para determinar la significancia entre variables histológicas se realizó la prueba de chi-cuadrado y la prueba exacta de Fisher. Se consideró estadísticamente significativa una $\mathrm{p}<0,05$.
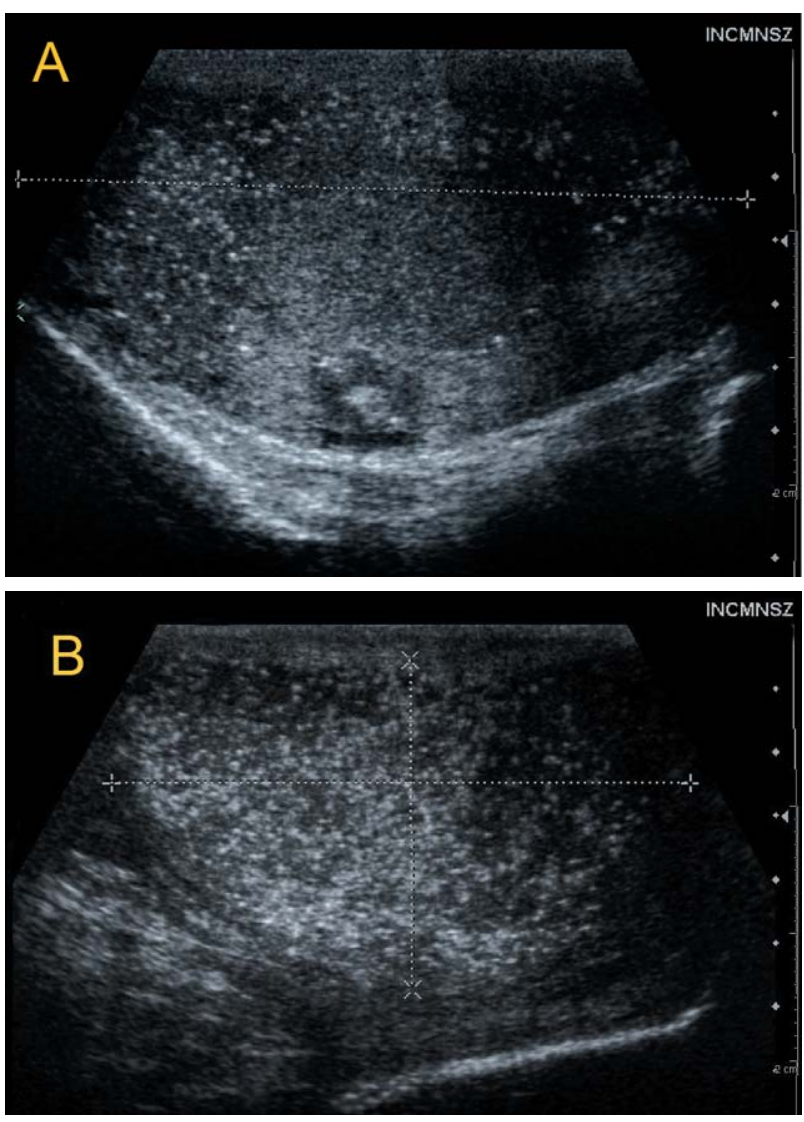

FIGURA 1. Ultrasonido testicular del mismo paciente con (A) MT además de microlitiasis intratumoral y (B) microlitiasis contralateral.

\section{RESULTADOS}

En la Tabla 1 se describen las diferencias entre los casos $\sin$ MT $(n=30,52,6 \%)$ y con MT $(\mathrm{n}=27,47,4 \%)$. Hubo 30 casos sin MT, de los cuales en $70 \%$ la histología correspondía a seminoma, el restante $30 \%$ a tumor germinal no seminomatoso (TGNS). Cinco casos $(17,7 \%)$ estaban en etapa II/III mientras 25 (83,3\%) estaban en estadio I. En ningún caso se documentó borde quirúrgico positivo ni invasión al cordón.

Se identificaron 27 pacientes con MT $(48,27 \%$ del total), seminoma en $44,4 \%$ y TGNS en el $55,6 \%$ restante. En cuanto al estadio el $51,8 \%$ se encontraban en estadio II/III $(29,6 \%$ en estadio II y $22,2 \%$ a estadio III) y el $48,2 \%$ en estadio I.

\section{ANÁLISIS ESTADÍSTICO}

No hubo diferencia estadística entre los grupos en cuanto a edad, lado afectado, requerimiento de tratamiento posterior, tamaño del tumor, recurrencia tumoral, ni tiempo de seguimiento. En 
las variables histológicas no hubo diferencia significativa en la incidencia de atrofia, neoplasia intratubular de células germinales (NITCG), invasión venosa/linfática ni a la albuginea.

Existió diferencia estadística en cuanto a tipo de tumor (mayor proporción de seminoma en el grupo sin MT y de TGNS en el grupo con MT), invasión al cordón espermático (mayor en el grupo con MT), incidencia de bordes quirúrgicos positivos (mayor en grupo con MT) $\mathrm{y}$ estadio (mayor estadio con MT) con $\mathrm{p}$ de 0,05, 0,048, 0,021 y 0,005 , respectivamente. Por tanto, la MT se asoció con TGNS, invasión al cordón espermático, borde quirúrgico positivo y estadio más avanzado. El otro grupo se asoció con seminoma, borde quirúrgico y cordón espermático negativo a neoplasia y estadio temprano.

En relación a la localización de la microlitiasis se identificaron 13 casos $(48,1 \%)$ con ella en ambos testículos, 12 en el testículo ipsilateral al tumor y dos en el contralateral (Tabla 2). Doce tenían microlitiasis intratumoral (5 bilateral y 7 ipsilateral al tumor). Únicamente fue significativo la mayor frecuencia de TGNS en el grupo con MT bilateral y de seminoma en el grupo de MT ipsilateral $(p=0,028)$. Las restantes variables no fueron significativamente diferentes entre los grupos.

\section{DISCUSIÓN}

La etiología de las calcificaciones no está completamente definida. Hay teorias que consideran que se tratan de acumulación de secreciones tubulares, degeneración celular dentro de los túbulos seminíferos o calcificaciones secundarias de las glicoproteínas secretadas en los túbulos ${ }^{1-4}$.
Tabla 1. Variables medidas entre grupos con y sin MT con la diferencia estadística entre ellos.

\begin{tabular}{|c|c|c|c|}
\hline & Sin MT (n=30) & Con MT $(n=27)$ & $\mathbf{p}$ \\
\hline Edad promedio (rango) & 32,28 (23 a 48) & 29,71 (19 a 46) & 0,098 \\
\hline Historia de Criptorquidia & 0 & 1 & 0,482 \\
\hline Meses evolución (rango) & $7,36(0,16$ a 36$)$ & $4,52(0,33$ a 24$)$ & 0,267 \\
\hline \multicolumn{4}{|l|}{ Lado } \\
\hline Derecho & 16 & 15 & \multirow[t]{2}{*}{0,920} \\
\hline Izquierdo & 11 & 11 & \\
\hline Tratamiento posterior & 17 & 21 & 0,143 \\
\hline \multicolumn{4}{|l|}{ Tipo de tumor } \\
\hline Seminoma & 21 & 12 & \multirow[t]{2}{*}{0,05} \\
\hline TGNS & 9 & 15 & \\
\hline \multicolumn{4}{|l|}{ Hallazgos patológicos } \\
\hline Atrofia & 6 & 5 & 0,841 \\
\hline Invasión a túnica vaginalis & 5 & 6 & 0,647 \\
\hline Invasión al cordón & 0 & 4 & 0,048 \\
\hline Invasión albuginea & 3 & 1 & 0,333 \\
\hline NITCG & 8 & 10 & 0,507 \\
\hline Borde quirúrgico positivo & 0 & 5 & 0,021 \\
\hline Tamaño (rango) & $5,85(0,16$ a 36$)$ & 6,04 (1 a 15) & 0,872 \\
\hline \multicolumn{4}{|l|}{ Estadio } \\
\hline I & 25 & 13 & \multirow[t]{2}{*}{0.005} \\
\hline II/III & 5 & 14 & \\
\hline Recurrencia & 5 & 4 & 0.546 \\
\hline $\begin{array}{l}\text { Seguimiento promedio } \\
\text { (rango) }\end{array}$ & $\begin{array}{c}44,63 \\
(0,43-122.3)\end{array}$ & $\begin{array}{c}39,26 \\
(1,9 \text { a } 179,3)\end{array}$ & 0,693 \\
\hline
\end{tabular}

Tabla 2. Variables medidas entre grupos con MT ipsilateral y bilateral con la diferencia estadística entre ellos.

\begin{tabular}{lccc}
\hline & Ipsilateral (n=12) & Bilateral (n=13) & $\mathbf{p}$ \\
\hline Lado & 7 & & \\
$\quad$ Derecho & 5 & 6 & 0,68 \\
$\quad$ Izquierdo & 10 & 9 & 0,363 \\
Tratamiento posterior & & & \\
Tipo & 8 & 3 & \\
$\quad$ Seminoma & 4 & 10 & $\mathbf{0 . 0 2 8 2}$ \\
$\quad$ TGNS & & & \\
Variables Histológicas & 2 & 3 & 0,541 \\
$\quad$ Atrofia & 3 & 3 & 0,636 \\
$\quad$ Invasión venosa/linfática & 2 & 1 & 0,46 \\
$\quad$ Invasión al cordón & 0 & 1 & 0,5357 \\
$\quad$ Invasión a T. Albuginea & 4 & 4 & 0,61 \\
$\quad$ NIT & 3 & 2 & 0,4277 \\
Borde quirúrgico positivo & & & \\
Estadio & $\mathbf{6}$ & 7 & 0,841 \\
$\quad$ I & $\mathbf{6}$ & 6 & 0,32 \\
$\quad$ Recurrencia & 1 & 3 &
\end{tabular}


En este análisis retrospectivo de pacientes con CaT, se encontró asociación de MT a TGNS (especialmente bilateral), borde quirúrgico positivo, estadio avanzado (II/III) y coexistencia en $47,3 \%$. Los pacientes con CaT sin MT se asociaron a seminoma, invasión a la albugínea, borde quirúrgico negativo y estadio I.

Este es el primer estudio que conocemos que investiga la relación que la MT tiene con el estadio clínico tumoral ${ }^{5,6}$. La mayoría de los reportes se han enfocado a este hallazgo en pacientes sin CaT y su relación al desarrollo posterior de neoplasias ${ }^{7-20}$. Estos han descrito una prevalencia promedio de $2,2 \%$ (441 de 20.404 pacientes sintomáticos) con un rango de $0,6 \%$ a $20,2 \%$, la mayor de ellas en pacientes sub-fértiles ${ }^{17,21}$. En promedio $20,1 \%$ (85/ 405) de los ultrasonidos con microlitiasis están asociados a un tumor testicular, estableciendo una clara asociación entre ambas ${ }^{18}$. Para apoyar esto Derogee et al. han corroborado la coexistencia significativa de estas entidades, sin embargo, este autor no encontró correlación de la histología del tumor germinal, con la localización ni con el tipo de calcificación ${ }^{13}$. Holm et al. estudió con biopsias la microlitiasis en el testículo contralateral al tumor e identificó que hay mayor frecuencia de NITCG en este y concluye que no se puede excluir la neoplasia intratubular en el testículo contralateral aun cuando no exista microlitiasis ${ }^{22}$.

Si bien hay publicaciones que reportan incidencias muy variables que van desde $6,02 \%$ al $74 \%$, la mayoría de ellas coinciden que la MT se asocia más frecuentemente a la presencia de neoplasia, esto puede representar sólo una manifestación del tumor y como se demostró en este estudio puede estar asociado a algunas características tanto de estirpe histológica como al estadio tumoral ${ }^{23,24}$.

\section{CONCLUSIÓN}

La presencia de MT en un paciente con tumor testicular puede estar asociada a TGNS, estadio avanzado y borde quirúrgico positivo. Se requieren estudios con un mayor número de pacientes para corroborar este hallazgo.

\section{REFERENCIAS}

1. Bieger RC, Passarge E, McAdams AJ. Testicular intratubular bodies. J Clin Endocrinol 1965;25:1340-1346.

2. Bunge RC, Bradbury JJ. Intratubular bodies of the human testis. J Urol. 1961;85:306-310.

3. Miller FN, Sidhu PS. Does testicular microlithiasis matter? A review. Clin Radiol 2002;57:883-890.
4. Vegni-Talluri M, Bigliardi E, Vanni MG, Tota G. Testicular microliths: their origin and structure. J Urol. 1980;124:105-107. Propeck et.al. Ultrasound Case of the Day. Radioghraphics 1993;13:693-695.

5. Grantham et al. Testicular neoplasms: 29 tumors studied by highresolution US. Radiology 1985;157:775-780

6. Woodward PJ, Sohaey R, O'Donoghue MJ, Green DE. Tumors and tumorlike lesions of the testis: radiologic-pathologic correlation. Radiographics 2002;22:189-216.

7. Hobarth K, Susani M, Szabo N, Kratzik C. Incidence of testicular microlithiasis. Urology 1992;40:464-467.

8. Ganem JP, Workman KR, Shaban SF. Testicular microlithiasis is associated with testicular pathology. Urology 1999;53:209-213.

9. Cast JEI, Nelson WM, Early AS, Biayani S, Cooksey G, Warnock NG et al. Testicular microlithiasis: prevalence and tumor risk in a population referred for scrotal sonography. AJR Am J Roentgenol 2000; 175:1703-1706.

10. Skyrme RJ, Fenn NJ, Jones AR, Bowsher WG. Testicular microlithiasis in a UK population: its incidence, associations and follow-up. BJU International 2000;86:482-485.

11. Bach AM, Hann LE, Shi WT, Yoo HH, Giess CS, Sheinfeld J et al. Testicular microlithiasis: what is its association with testicular cancer? Radiology 2001;220:70-75.

12. Otite U, Webb JA, Oliver RT, Badenoch DF, Nargund VH. Testicular microlithiasis; is it a benign condition with malignant potential? Eur Urol. 2001;40:538-542

13. Derogee M, Bevers RF, Prins HJ, Jonges TG, Elbers FH, Boon TA. Testicular microlithiasis, a premalignant condition: prevalent histopathologic findings, and relation to testicular tumor. Urology 2001;57:1133-1137.

14. Peterson AC, Bauman JM, Light DE, McMann LP, Costabile RA. The prevalence of testicular microlithiasis in an asymptomatic population of men 18 to 35 years old. J Urol. 2001;166:2061-2064.

15. Middleton WD, Teefey SA, Santillan CS. Testicular microlithiasis: prospective analysis of prevalence and associated tumor. Radiology 2002;224:425-428

16. Leenen A, Riebel T. Testicular microlithiasis in children: sonographic features and clinical implications. Pediatr Radiology 2002;32:575-579.

17. de Gouveia Brazao CA, Pierik FH, Oosterhuis JW, Dohle GR, Looijenga LH, Weber RF (2004) Bilateral testicular microlithiasis predicts the presence of the precursor of testicular germ cell tumors in subfertile men. J Urol. 2004;171:158-160.

18. Miller FNAC, Roairo S, Clarke JL, Spriprasad S, Muir GH, Sidhu PS. Testicular calcification and microlithiasis: association with primary intra-testicular malignancy in 3,477 patients. Eur Radiol. 2007;17: 363-369.

19. Rashid HH, Cos LR, Weinberg E, Messing EM. Testicular microlithiasis: a review and its association with testicular cancer. Urologic Oncology 2004;22:285-289.

20. Parenti GC, Zago S, Lusa M, Campioni P, Mannella P. Association between testicular microlithiasis and primary malignancy of the testis: our experience and review of the literature. Radiol Med (Torino). 2007;112(4):588-596

21. Sakamoto H, Shichizyou T, Saito K, Okumura T, Ogawa Y, Yoshida H, Kushima M. Testicular microlithiasis identified ultrasonographically in japanese adult patients: prevalence and associated conditions. Urology 2006;68:636-641

22. Holm M, Hoei-Hansen CE, Rajpertde Meyts E, Skakkebaek NE. Microcalcifications and carcinoma in situ of the testis. BJU International 2001;87(2):144-149.

23. Rodríguez-Villalba R, Scarfini M, Ruiz-Castañé E, Pomerol-Monseny JM. Testicular microlithiasis: Does exist any relationship with malignant tumour? Actas Urol. Esp 2004;28(1):38-39.

24. Ikinger U, Wurster K, Terwey B. Microcalcifications in testicular malignancy. Urology 1982;19:525-258.

Correspondencia autor: Dr. M. Villalobos Gollás Departamento de Urología. Instituto Nacional de Ciencias Médicas y Nutrición "Salvador Zubirán"

Vasco de Quiroga, 15, Col. Secc. XVI, Tlalpan, 14000 México D.F. Tel.: (52)55-5487-0900 ext. 2260

E-mail autor: m.villalobos.gollas@gmail.com

Información artículo: Original - Cáncer de testículo

Trabajo recibido: junio 2007

Trabajo aceptado: julio 2007 